\title{
An evaluation of the British Pharmacopoeial assay of heparin: a comparison with other methods
}

\author{
GA SHAH, TZ DHALL, I FERGUSON, MR DAVIS, DT GRAHAM, AR POMEROY, \\ AND DP DHALL
}

From the Vascular Laboratory and Pharmacy, Woden Valley Hospital, Garran, ACT, and the National Biological Standards Laboratory, Canberra, Australia

SUMMARY The potencies of six commerically manufactured heparins have been measured by the British Pharmacopoeial (BP) assay and activated partial thromboplastin time (APTT), protamine sulphate, and anti-Xa assays. The APTT/BP potency ratios were found to vary with the preparation but this was not dependent on the tissue source of heparin. For mucosal heparins, the anti-Xa/BP potency ratios were close to unity, but for heparin of lung origin the anti-Xa potency was approximately one-quarter of the BP potency. Four heparin fractions prepared by column gel chromatography of a commerical heparin were similarly examined by all four assays, and there was a wide divergence between the BP potency estimates and those obtained with the other methods. The degree of divergence was found to depend on the molecular size of the fraction.

Heparin is used widely in low-dose regimes for prophylaxis and in therapeutic doses for the treatment of established venous thromboembolism. In its antithrombotic role, heparin is used to potentiate an anti-Xa action of antithrombin III. The mode of action of heparin as an anticoagulant is less clear, and, when used in this way, the risk of haemorrhage is monitored by clotting assays such as the activated partial thromboplastin time (APTT). As generally marketed, heparin is standardised with a pharmacopoeial technique such as the British Pharmacopoeial (BP) assay. Since the activity of commerically manufactured heparin varies with the tissue source of heparin, with the molecular weight composition, and the assay method, the use of one pharmacopoeial method to characterise the varying roles of heparin (antithrombotic and anticoagulant) is open to question. In this investigation several heparins manufactured commercially (from lung and mucosa) have been assayed by a variety of techniques to provide information on the adequacy of the BP assay to measure the activity of unfractionated heparins. In addition, heparin fractions separated from one commerical preparation have been similarly examined.

Received for publication 16 November 1979

\section{Material and methods}

HEPARINS

A bovine mucosal sodium heparin was used as a reference heparin. It was standardised by the BP assay against the Third International Standard Heparin by the National Biological Standards Laboratory, Australia. The specific activity of the reference heparin was found to be 146 units per $\mathrm{mg}$ and it was used in assaying the following five mucosal and one lung preparations obtained from various manufacturers.

Heparin of mucosal origin was obtained from: Allen and Hanburys (Glaxo Australia)

$1000 \mathrm{U} / \mathrm{ml}$ Batch No 251324 (Boronia, Australia)

Weddel Pharmaceuticals Limited

$1000 \mathrm{U} / \mathrm{ml}$ Batch No 642A (London, UK)

Evans Medical Limited

$1000 \mathrm{U} / \mathrm{ml}$ Batch No 5N083A (Liverpool, UK)

The Boots Company Limited

$25000 \mathrm{U} / \mathrm{ml}$ Batch No 5456 (Nottingham, UK)

Commonwealth Serum Laboratories

$5000 \mathrm{U} / \mathrm{ml}$ Batch No 621.02-151/2 (Melbourne,

Australia)

Heparin of lung origin was obtained from:

The Upjohn Company

$10000 \mathrm{U} / \mathrm{ml}$ Batch No 819EX (Michigan, USA) 562 
HEPARIN ASSAYS

The British Pharmacopoeial assay ${ }^{1}$ was modified according to Walton and Wright, ${ }^{2}$ and the clotting time endpoint was determined using a coagulometer. This modification of the BP method provides an objective measure of the endpoint and does not alter the potency estimate. The APTT was measured with activated Thrombofax (Ortho Diagnostics, New Jersey, USA) according to the instructions of the manufacturer. The anti-Xa assay was performed according to the method of Denson and Bonnar ${ }^{3}$ with a commercially available heparin assay kit (Diagnostic Reagents, Thame, Oxon, UK). In the protamine titration assay, 4 the concentration of protamine sulphate (Boots, Nottingham, UK) was increased in steps of $2 \mathrm{mg} / \mathrm{dl}$ (instead of $5 \mathrm{mg} / \mathrm{dl}$ ) to enhance the sensitivity of the assay. Thrombin (Parke Davis, Detroit, USA) concentrations were chosen to give a thrombin clotting time with unheparinised plasma of 10-12 seconds.

Plasma was prepared from blood obtained from 12 normal blood donors. Blood (9 volumes) was collected into $3.8 \%$ sodium citrate (1 volume) and centrifuged at $1300 \mathrm{~g}$ for 15 minutes. The platelet poor plasma (PPP) was pooled and stored in vials at $-77^{\circ} \mathrm{C}$. Heparinised plasma was prepared by adding 1 volume of heparin to 9 volumes of PPP. All clotting assays were performed manually using

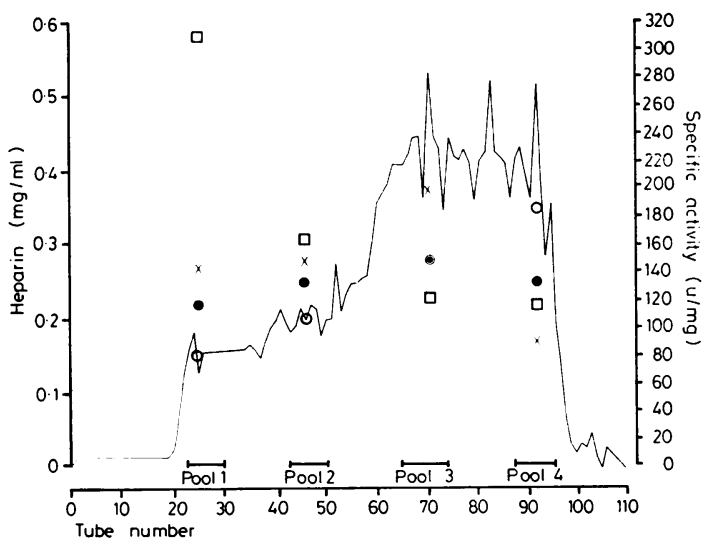

Elution profile for Allen and Hanburys heparin. The concentration of heparin in each fraction was measured by a toluidine blue metachromatic assay (scale on left). Fractions corresponding to the major areas of the elution curve were collected into four pools as shown. The mean molecular size of heparin fractions declines from pool 1 to pool 4. The specific activity of heparin in each pool was measured by BP assay ( $\square)$, anti-Xa assay $(\bigcirc)$, protamine titration $(\bigcirc)$, and $A P T T$ $(\times)$ (scale on right). siliconised glass tubes at $37^{\circ} \mathrm{C}$. Plastic or siliconised glassware was used throughout.

Calibration curves with the reference heparin were determined for each method. For the anti-Xa assay a $\log /$ linear relationship was established between the clotting time and the concentration of heparin in plasma. A linear relationship was obtained between heparin concentration and the amount of protamine needed to neutralise it. For the APTT assay, a linear relationship between heparin concentration and the clotting time followed an initial non-linear curve. For this assay, test heparin concentrations on the linear portion of the calibration curve were selected.

Using each of the four techniques, heparin activity was determined from the calibration curves in units per $\mathrm{ml}$. To derive specific activity of heparin preparations a metachromatic assay ${ }^{5}$ was used to measure the concentration of heparin in $\mathrm{mg} / \mathrm{ml}$. In this technique the absorbance at $505 \mathrm{~nm}$ of heparinAzure A mixture was measured with a Unicam SP1800 spectrophotometer. A calibration curve was established with the reference heparin (lyophilised powder), and dilutions of test heparins were chosen to lie where the absorbance at $505 \mathrm{~nm}$ linearly related to the concentration of heparin.

\section{HEPARIN FRACTIONATION}

Five millilitres of the heparin manufactured by Allen and Hanburys was fractionated by gel filtration on a $2.5 \times 45 \mathrm{~cm}$ column of medium grade Sephadex G50. The heparin was eluted with distilled water at a flow rate of $36 \mathrm{ml} / \mathrm{h}$. The heparin content in the elution fractions was monitored with a separate metachromatic technique using toluidine blue. For each $1 \mathrm{ml}$ fraction, heparin content was measured by mixing $50 \mu \mathrm{l}$ of the fraction with $3 \mathrm{ml}$ of an aqueous solution of $0.0025 \% \mathrm{w} / \mathrm{v}$ toluidine blue and measuring the transmittance at $500 \mathrm{~nm}$ relative to that for known concentrations of the reference heparin preparation. Fractions corresponding to major areas of the elution curve were collected into four pools (Figure) and the specific activity of each pool was determined by the four assays.

\section{Results}

The specific activities of all heparin preparations measured by each of the four techniques as well as the coefficient of variation are shown in Table 1a. The coefficient of variation derived from 15 measurements on the Allen and Hanburys heparin preparation is largest for the anti-Xa method and least for the BP assay using the coagulometer. It can be seen that the specific activity varies with the pre- 
Table 1(a) Specific activities (U/mg) of heparins tested by each of the four techniques

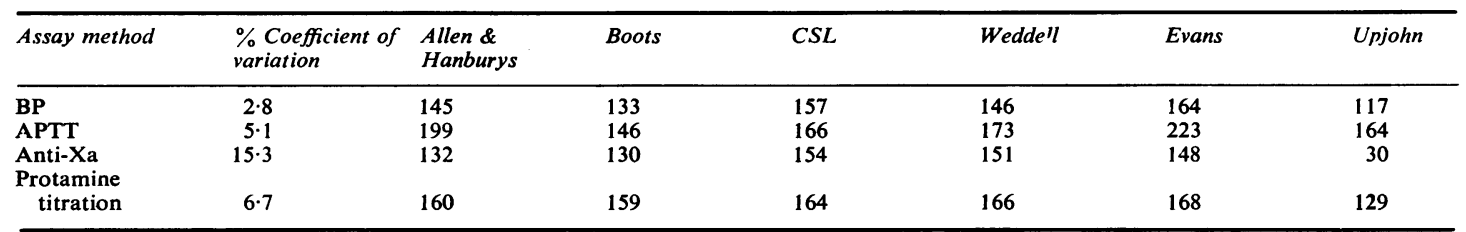

The specific activity of the reference heparin was $146 \mathrm{U} / \mathrm{mg}$. The results are the mean of two measurements except for the Allen and Hanburys heparin where each result is the mean of 15 measurements. The coefficient of variation is derived from the data for the Allen and Hanburys heparin. The heparins are of mucosal origin with the exception of Upjohn heparin, which is of lung origin.

(b) BP potency of each heparin tested expressed as a percentage of the labelled potency $(U / \mathrm{ml})$

\begin{tabular}{lccccrr}
\hline Assay method & $\begin{array}{l}\text { Allen \& } \\
\text { Hanburys }\end{array}$ & Boots & CSL & Weddell & Evans & Upjohn \\
\hline BP & 104 & 91 & 104 & 96 & 107 & 92 \\
APTT/BP ratio & 1.38 & $1 \cdot 10$ & 1.06 & 1.19 & 1.36 & 0.89 \\
Anti-Xa/BP ratio & 0.91 & 0.98 & 0.98 & 1.03 & 1.02 & 1.11 \\
Protamine titration/BP ratio & 1.11 & 1.20 & 1.05 & 0.14 & 0.66 \\
Anti-Xa/APTT ratio & 0.66 & 0.89 & 0.93 & 0.87 & 0.19 \\
\hline
\end{tabular}

The potencies measured with the APTT, anti-Xa and protamine titration assays are expressed as a ratio of the BP potency. The anti-Xa/ APTT ratios are also shown.

paration and with the method of assay. The results are the mean of two measurements except for those of the Allen and Hanburys heparin which are the mean of 15 measurements.

In Table $1 \mathrm{~b}$ the potencies of all heparins measured by the BP assay are given as a percentage of the labelled potency (units/ml). The potencies obtained by the other assays are expressed relative to the BP potency. The APTT/BP ratios varied from 1.06 for heparin by Commonwealth Serum Laboratories to 1.40 for Upjohn heparin. This variation appeared to be unrelated to the tissue source of the preparation. For the mucosal heparins the anti-Xa/BP ratios were close to unity, while for the Upjohn lung heparin the anti-Xa potency was a quarter of the BP potency. The potencies determined by the protamine titration assay were generally in agreement with the BP potencies, although the protamine titration/BP ratio was significantly greater than unity for Boots heparin. The anti-Xa/APTT ratio varied from 0.66 to 0.93 for the mucosal preparations while it

Table 2 Four heparin pools derived from fractionation of Allen and Hanburys heparin assayed by the BP, $A P T T$, anti-Xa and protamine titration methods

\begin{tabular}{lllll}
\hline Assay method & Pool 1 & Pool 2 & Pool 3 & Pool 4 \\
\hline APTT/BP ratio & 0.46 & 0.91 & 1.56 & 0.74 \\
Anti-Xa/BP ratio & 0.26 & 0.66 & 1.24 & 1.55 \\
Protamine titration/BP ratio & 0.38 & 0.82 & 1.24 & 1.11 \\
Anti-Xa/APTT ratio & 0.56 & 0.72 & 0.80 & 2.08 \\
\hline
\end{tabular}

The results are expressed as a ratio of the value obtained by the $\mathrm{BP}$ assay. Anti-Xa/APTT ratios for the four pools are also shown. These ratios are derived from the specific activities shown in the Figure. was only 0.19 for the lung heparin.

The specific activities of the four pools obtained by fractionating Allen and Hanburys heparin were determined by the four assays and these are shown in the Figure. It can be seen that the specific activities measured for the four pools depend upon the assay method and on the molecular size of the fraction. Table 2 shows the measured potencies for these four pools relative to the BP potency as well as the anti-Xa/APTT ratio. For pool 1 the heparin potency obtained by the BP method is more than twice those obtained by the other methods. For pools 2 and 3, the potency ratios (relative to the BP method) show a marked increase as the molecular size decreases. The anti-Xa/BP ratio showed a further increase in pool 4 while the APTT/BP ratio showed a dramatic decrease. This is reflected in the anti-Xa/APTT ratio which was 2.08 for pool 4 (the smallest molecular size pool) but less than 1 for the other pools.

\section{Discussion}

Commercially marketed heparins are assayed against a pharmacopoeial assay. In the investigations described, we have examined six commercial heparins with a variety of assays. Although significant variations were found in the six heparins tested using the BP method, all the measured potencies (including that of the Upjohn heparin, which complies with the requirements of the United States Pharmacopoeia) were within the tolerance limits specified in the British Pharmacopoeia. ${ }^{1}$ 
Investigations descrited in this study show that the assigned potency of commercial heparins can vary according to the assay used and that those obtained with the BP assay are not necessarily representative of the values obtained with other methods. The APTT/BP potency ratios varied considerably with the preparation but this was found not to depend on the tissue source of heparin. In four of the six heparins tested, the APTT method gave a higher measurement of heparin than did the BP assay. On the other hand, when the BP assay was compared with the anti-Xa method, good agreement was obtained for the heparins of mucosal origin. For the lung heparin, the potency measured with the anti-Xa assay was found to be markedly reduced. This is reflected in the difference between the anti-Xa/ APTT potency ratios for lung and mucosal heparins. It has been shown previously ${ }^{6}$ that this ratio was less than 1.0 for lung and greater than 1.0 for mucosal heparin.

Differences between the estimates obtained by the BP method and the other assays were even more pronounced when the various assays were tested against fractions of heparin. Higher potency values of the large molecular size fractions were found using the BP method than with the other methods. Conversely, the small molecular size fractions gave higher assay values with the other methods. The discrepancies between the potency estimates using the BP method and the other clinical methods observed in this investigation may be due in part to the use of animal blood ${ }^{7}$ in the BP assay. The anti-Xa/ APTT ratio varied with the molecular size of heparin fraction and was dramatically raised in the fraction with the lowest molecular size.

A number of studies have shown that, after parenteral administration, heparin-induced prolongation in the APTT is similar regardless of the tissue source. ${ }^{8-11}$ In contradistinction, the difference between the lung and mucosal heparins in potentiating anti-Xa activity in vitro is also clearly demonstrable in vivo after injection into human subjects. ${ }^{6}$

Since the APTT potencies of the lung and mucosal heparins are similar, both in vitro and in vivo, the large difference in the anti-Xa/APTT ratio between the two sources of heparins originates predominantly in their anti-Xa potencies. This finding may be of some relevance to the current clinical practice of heparin administration in low dosage for its antithrombotic action. It has been assumed ${ }^{6}$ that, for optimal antithrombotic prophylaxis, heparin with a high anti-Xa/APTT ratio is more desirable than one with a lower ratio since such a preparation might confer enhanced protection for an equivalent risk of haemorrhage. However, it has been shown in the $\operatorname{dog}^{12}$ that mucosal heparin causes significantly greater 'delayed haemorrhage' than lung heparin and that protamine reversal is significant only with lung heparin. It seems that only the APTT potency of the two heparins is capable of being reversed completely, and a significant fraction of the initial anti-Xa potency remains unneutralised. ${ }^{13}$ It is possible that the unneutralised anti-Xa activity underlies the failure of protamine to reduce effectively the incidence of 'delayed haemorrhage' with heparin of mucosal origin. Thus, the possibility remains that the use of a heparin preparation with a high anti-Xa/APTT ratio may also be associated with an enhanced risk of haemorrhage.

\section{References}

${ }_{1}^{1}$ British Pharmacopoeia. London: HMSO, 1973.

2 Walton PL, Wright BM. An automatic coagulometer. J Physiol (Lond) 1964;172:7p. (Abstract)

${ }^{3}$ Denson KWE, Bonnar J. The measurement of heparin. A method based on the potentiation of anti-Factor Xa. Thromb Diath Haemorrh 1973;30:471-9.

4 Dacie JV, Lewis SM. Practical Haematology. Edinburgh and London: Churchill Livingstone, ELBS edition, 1974.

5 Jaques LB, Wollin A. A modified method for the colorimetric determination of heparin. Can $J$ Physiol Pharmacol 1967;45:787-94.

${ }^{6}$ Barrowcliffe TW, Johnson EA, Eggleton CA, Thomas DP. Anticoagulant activities of lung and mucous heparins. Thromb Res 1978;12:27-36.

7 Shen LL, Barlow GH, Holleman WH. Differential activities of heparins in human plasma and in sheep plasma. Effects of heparin molecular sizes and sources. Thromb Res 1978;13:671-9.

${ }^{8}$ Gomez-Perez F. Anticoagulant activity of two commercially available heparin preparations. A controlled study. J Clin Pharmacol 1972;12:413-6.

9 Baltes BJ, Diamond S, D'Agostino RJ. Comparison of anticoagulant activity of two preparations of purified heparin. Clin Pharmacol Ther 1973;14: 287-90.

${ }^{10}$ Silverglade A. Biological equivalence of beef lung and hog mucosal heparins. Curr Ther Res 1975;18:91-103.

11 Jain AK, McMahon FG, Ryan JR. Comparison of anticoagulant activity of three preparations of heparin. Curr Ther Res 1977;22:427-33.

12 Abbott WM, Warnock DF, Austen WG. The relationship of heparin source to the incidence of delayed hemorrhage. J Surg Res 1977;22:593-7.

${ }^{13}$ Michalski R, Lane DA, Pepper DS, Kakkar VV. Neutralization of heparin in plasma by platelet Factor 4 and protamine sulphate. $\mathrm{Br} J$ Haematol $1978 ; 38: 561-71$.

Requests for reprints to: Dr DP Dhall, Surgical Unit Woden Valley Hospital, PO Box 11, Woden, ACT 2606 